\title{
UROLOGICAL ONCOLOGY
}

Pathological Outcomes and Biochemical Progression in Men with T1c Prostate Cancer
Undergoing Radical Prostatectomy with Prostate Specific Antigen 2.6 to 4.0 vs 4.1 to 6.0 $\mathrm{ng} / \mathrm{ml}$ Makarov DV, Humphreys EB, Mangold LA, Walsh PC, Partin AW, Epstein JI, Freedland SJ

James Buchanan Brady Urological Institute, The Johns Hopkins Medical Institutions, Baltimore, Maryland, USA J Urol. 2006; 176: 554-8

Purpose: Recent studies have suggested that the cut point for recommending prostate biopsy among men with a normal digital rectal examination should be greater than $2.5 \mathrm{ng} / \mathrm{ml}$ as opposed to the more traditional greater than $4.0 \mathrm{ng} / \mathrm{ml}$. We compared outcomes between men with clinical stage T1c disease undergoing radical prostatectomy who had a low vs slightly increased prostate specific antigen.

Materials and Methods: The study population consisted of 2,896 men treated with radical prostatectomy between 1985 and 2004 at a tertiary care referral center with clinical stage T1c disease and a pre-biopsy prostate specific antigen between 2.6 and $6.0 \mathrm{ng} / \mathrm{ml}$. Using multivariate analysis we evaluated the association between prebiopsy prostate specific antigen 2.6 to $4.0 \mathrm{ng} / \mathrm{ml}(784)$ vs 4.1 to $6.0 \mathrm{ng} / \mathrm{ml}(2,112)$, and pathological outcomes and biochemical progression.

Results: After adjusting for multiple clinical and pathological characteristics, lower preoperative serum prostate specific antigen values were associated with decreased odds of Gleason score 7 or greater in the surgical specimen $(\mathrm{p}=0.004)$, positive surgical margins $(\mathrm{p}=0.02)$ and extraprostatic extension $(\mathrm{p}=0.001)$. There was no significant association between these preoperative prostate specific antigen groups and odds of seminal vesicle invasion $(\mathrm{p}=0.47)$ or lymph node metastasis $(\mathrm{p}=0.90)$. Among the 1,534 men with followup information available there was a trend for increased risk of biochemical progression associated with a higher preoperative prostate specific antigen, although this trend did not reach statistical significance (relative risk $1.48,95 \%$ CI $0.69-3.19, \mathrm{p}=0.31)$.

Conclusions: In the current study of men with clinical stage T1c treated with radical prostatectomy a lower preoperative prostate specific antigen was associated with significantly more favorable pathological findings. Whether this degree of improved outcomes justifies the limitations associated with decreasing the prostate specific antigen cut point (e.g. increased biopsies performed and diagnosis of insignificant cancers) remains to be determined.

\section{Editorial Comment}

The authors focus on a rather large series of patients with a low serum PSA and biopsy-confirmed prostate cancer undergoing radical prostatectomy. Indeed, 784 patients with a PSA between 2.6 and $4.0 \mathrm{ng} / \mathrm{mL}$ were compared to patients with a PSA between 4.1 and $6.0 \mathrm{ng} / \mathrm{mL}$. I wonder how suspicion of prostate cancer was generated in the first group, e.g. by abnormal digital examination?

Nevertheless, the results are interesting and give support to the notion that prostate cancer is an aggressive disease, even with low PSA. Positive surgical margins and capsular penetration were found in $6 \%$ and $14 \%$, respectively, in the first group and in $9 \%$ and $21 \%$ in the higher PSA group. Recurrence-free survival was inferior after 10 years in the elevated PSA group.

What does that mean for the practicing urologist? To my opinion: detect and treat prostate cancer as early as possible.

Dr. Andreas Bohle

Professor of Urology

HELIOS Agnes Karll Hospital

Bad Schwartau, Germany 


\section{Long-Term Followup of a Randomized Study of Locally Advanced Prostate Cancer Treated with Combined Orchiectomy and External Radiotherapy versus Radiotherapy Alone}

Granfors T, Modig H, Damber JE, Tomic R

Department of Urology, Central Hospital, Vasteras, Sweden

J Urol. 2006; 176: 544-7

Purpose: In a randomized study we compared the combination of orchiectomy and radiotherapy to radiotherapy alone as treatment for locally advanced prostate cancer. Patients who were treated only with radiotherapy initially underwent castration therapy at clinical progression, providing the opportunity to compare immediate vs deferred endocrine intervention.

Materials And Methods: In this prospective study 91 patients with locally advanced prostate cancer were randomized to receive external beam radiotherapy (46) or combined orchiectomy and radiotherapy (45) after surgical lymph node staging. Survival rates were calculated.

Results: During 14 to 19 years of followup $87 \%$ of the patients in the radiotherapy group and $76 \%$ in the combined orchiectomy and radiotherapy group died $(\log$ rank $\mathrm{p}=0.03)$. Prostate cancer mortality was $57 \%$ and $36 \%$, respectively ( $\log$ rank $\mathrm{p}=0.02$ ). The difference in favor of combined treatment was mainly caused by lymph node positive tumors. For node negative tumors there was no significant difference in the survival rates. Conclusions: Immediate androgen deprivation should be considered instead of deferred endocrine treatment started at clinical progression for prostate cancer with spread to regional lymph nodes. While awaiting evidence from randomized trials, one should consider full dose radiotherapy for local control of locally advanced prostate cancer even when it is lymph node positive.

\section{Editorial Comment}

This paper gives the long-term results of a simple but well-done trial: immediate or deferred hormone ablative treatment in patients undergoing external beam radiation therapy (ERBT) after surgical lymph node staging.

The answer is clear-cut: immediate hormone ablative therapy is better than deferred therapy with regard to survival. This difference was most predominant in lymph node positive patients. In conclusion, these data and other papers strongly support the use adjuvant endocrine treatment in radiotherapy against prostate cancer.

Dr. Andreas Bohle

Professor of Urology HELIOS Agnes Karll Hospital Bad Schwartau, Germany

\section{NEUROUROLOGY \& FEMALE UROLOGY}

\section{Transurethral Radiofrequency Energy Collagen Micro-Remodeling For the Treatment of Female Stress Urinary Incontinence}

Appell RA, Juma S, Wells WG, Lenihan JP, Klimberg IW, Kanellos A, Reilley SF

Department of Urology, Baylor College of Medicine, Houston, Texas, USA

Neurourol Urodyn. 2006; 25: 331-6

Aims: This prospective, randomized, controlled clinical trial was performed to demonstrate the 12 months safety and efficacy of transurethral radiofrequency energy (RF) collagen micro-remodeling in women with stress urinary incontinence (SUI). 